\title{
CLDN5 affects IncRNAs acting as ceRNA dynamics contributing to regulating blood-brain barrier permeability in tumor brain metastasis
}

\author{
SHUN-CHANG MA ${ }^{1,2^{*}}$, QI LI ${ }^{3 *}$, JIA-YI PENG ${ }^{1}$, JIAN-LONG ZHOUWEN ${ }^{1}$, DAI-NAN ZHANG ${ }^{1}$, \\ CHUAN-BAO ZHANG ${ }^{1}$, WEN-GUO JIANG ${ }^{4}$ and WANG JIA ${ }^{1}$ \\ ${ }^{1}$ Department of Neurosurgery, Beijing Tiantan Hospital; ${ }^{2}$ Department of Neurosurgery, Fuxing Hospital; \\ ${ }^{3}$ Core Laboratory for Clinical Medical Research, Beijing Tiantan Hospital, Capital Medical University, \\ Beijing 100050, P.R. China; ${ }^{4}$ Metastasis and Angiogenesis Research Group, University Department of Surgery, \\ Cardiff University School of Medicine, Heath Park, Cardiff CF14 4XN, UK
}

Received July 12, 2017; Accepted January 4, 2018

DOI: $10.3892 /$ or.2018.6208

\begin{abstract}
The blood-brain barrier (BBB) constitutes an efficient organization of tight junctions that limits the delivery of tumor to the brain. The principal tight junction protein in BBB is claudin-5 (CLDN5), but its mechanism of action remains largely unknown. Long non-coding RNAs (lncRNAs) are aberrantly expressed in many cancers, some lncRNAs play key roles in regulating $\mathrm{BBB}$ permeability and are involved in tumor brain metastasis. In particular, lncRNAs can function as competing endogenous RNAs (ceRNAs). Herein, we investigated whether ceRNA dysregulation is associated with alterations of the level of CLDN5 in human brain vascular endothelial hCMEC/D3 cells. The Affymetrix Human Transcriptome Array 2.0 and Affymetrix GeneChip miRNA 4.0 Array were used to detect the expression levels of 2,578 miRNAs, 22,829 lncRNAs, and 44,699 mRNAs in pLL3.7-CLDN5-transfected and pLL3.7 control hCMEC/D3 cells. The distinctly expressed miRNAs, lncRNAs, and mRNAs were subjected to construction of miRNA-lncRNA-mRNA interaction network. A total of 41 miRNAs, 954 lncRNAs, and 222 mRNAs were found to be differentially expressed between the CLDN5-overexpressing and control group. 148 lncRNA acting as ceRNAs were identified based on the miRNA-lncRNA-mRNA interaction network. The function of differential mRNA in the network was determined by GO and pathway analysis. The potential roles of the 27 ceRNAs were revealed, the possible biology functions of these regulatory ceRNAs mainly included tight junction, focal
\end{abstract}

Correspondence to: Professor Wang Jia, Department of Neurosurgery, Beijing Tiantan Hospital, Capital Medical University, 6 Tiantan Xili, Beijing 100050, P.R. China

E-mail: jwttyy@126.com

*Contributed equally

Key words: CLDN5, blood-brain barrier, IncRNA, ceRNA, biological function adhesion, cell-cell adhesion, cell growth and apoptosis. The identified sets of miRNAs, lncRNAs and mRNAs specific to CLDN5-overexpressing hCMEC/D3 cells were verified by quantitative real-time RT-PCR experiment. Our study predicts the biological functions of a multitude of ceRNAs associated with the alteration of CLDN5 in brain vascular endothelial cells. Our data suggest that these dysregulated ceRNAs, in conjunction with the high CLDN5 levels, could serve as useful targets of prevention of brain metastasis formation. Further studies are warranted to determine the role of these ceRNAs in facilitating the function of CLDN5 in brain-tumor barrier.

\section{Introduction}

The blood-brain barrier (BBB) is located at the level of cerebral capillaries in the forefront of the defense line of the central nervous system (CNS) and restricts the flow of essential components into and out of the CNS $(1,2)$. The most important cellular elements of the BBB consist of endothelial cell, astrocyte, pericyte, and the adjacent neurons (3). The endothelial cells are connected by junction complex, in which tight junctions (TJs) play a significant role. Key components of the TJs are the transmembrane protein, and the best characterized TJs in cerebral endothelial cells are occluding, claudins and junctional adhesion molecules (JAMs) (4).

Brain metastases constitute a significant part of intracranial tumors. The majority of brain metastases originate from lung cancer, breast cancer and malignant melanoma (5). Metastatic cells invading the CNS parenchyma, however, have to pass the BBB. Since brain metastases represent a great therapeutic challenge, it is important to understand the mechanisms of the interaction of tumor cells with the BBB to find targets of prevention of brain metastasis formation.

The attachment of tumor cells to brain endothelial cells and the transendothelial migration of tumor cells are the key step in brain metastasis, plenty of molecules and signal pathways participated in this complicated process. Claudins are small proteins (20-27 kDa) (6), and the principal claudin in brain endothelial cells is claudin-5 (CLDN5) (7). CLDN5 forms the backbone of 
the tight junction and was shown to be solely responsible for the paracellular barrier that exists between epithelial cell, and absence of CLDN5 leads to a selective opening of the BBB to molecules smaller than $800 \mathrm{Da}(8)$. CLDN5 has also recently been shown to directly play a role in the interaction of metastatic tumor cells and brain endothelial cells (9), but its mechanism of action remains largely unknown.

Recent studies have described a complicated interplay among diverse RNA molecules, including coding and non-coding RNAs $(10,11)$. Human genome generates plurality of regulatory RNAs that are either long non-coding RNAs (lncRNAs) or small non-coding RNA such as microRNAs (miRNAs). LncRNAs are defined as $>200$ nucleotides and unable to be translated into proteins. Accumulating data show that lncRNAs are major regulators of physiological and disease-related gene expression through various mechanisms. The dysregulated lncRNA expression has been documented in various disease states, and their tissue specificity makes them attractive candidates as diagnostic or prognostic biomarkers or therapeutic agents. miRNAs are endogenous 22 nucleotide RNAs, which post-transcriptionally regulate the gene expression through interaction between their $5^{\prime}$ end and the $3^{\prime}$ untranslated region (UTR) of mRNA. The functions of miRNA have been elucidated extensively and it participates in regulating a variety of cellular events (12-14). Recent studies indicated that some specific lncRNAs can serve as competitive endogenous RNAs (ceRNA) to control miRNAs available for binding with targets, functionally sequester miRNAs and as miRNA sponges, thereby alleviating the inhibitory effect of miRNA on their respective mRNA targets (15). The function introduces an extra layer of complexity in the miRNA-target interaction network. The complexity and diversity of potential ceRNA interactions have been described with the identification of abundant lncRNAs. Understanding this novel RNA interaction will lead to significant insight into gene regulatory networks in a variety of cell process (16-19).

The involvement of ceRNA regulation as a factor of CLDN5 modulation in brain vascular endothelial cells has not been previously investigated. To gain further understanding of how CLDN5 mediates its activities in tumor brain metastasis, herein, in this study, we examined changes in globe mRNA, miRNA, IncRNA gene expression when CLDN5 was overexpressed in the human brain vascular endothelial cell line, hCMEC/D3. We have identified a number of ceRNAs whose expression levels were altered as a consequence of high CLDN5 expression. The identified sets of lncRNA, miRNA and mRNA specific to CLDN-5-overexpressing hCMEC/D3 cells were subsequently confirmed by quantitative reverse transcription-polymerase chain reaction (qRT-PCR).

\section{Materials and methods}

Cell culture and transduction. Human brain vascular endothelial hCMEC/D3 cells were obtained from Institut Cochin (Université René Descartes, Paris, France). Cells were cultured in the EBM-2 medium supplemented with EGMTM-2 Bullet kit (Lonza, Walkersville, NJ, USA) in a humidified $37^{\circ} \mathrm{C}$ incubator with an atmosphere of $5 \% \mathrm{CO}_{2}$.

In the overexpression experiment, the CLDN5 expression vector was constructed by inserting a human CLDN5 cDNA into pLL3.7-GFP. CLDN5-pLL3.7 or control pLL3.7 vector were transfected into 293T cells using Lipofectamine 3000 (Thermo Fisher Scientific, Inc., Waltham, MA, USA). Supernatant containing retroviruses was collected and concentrated 48 and $72 \mathrm{~h}$ later and was used for the transduction. The hCMEC/D3 cells were transducted with the virus-containing medium plus $8 \mu \mathrm{g} / \mathrm{ml}$ polybrene. Forty-eight hours after beginning the transduction, $\mathrm{GFP}^{+}$cells were sorted and the expression of CLDN5 was confirmed by qRT-PCR.

RNA extraction. Total RNA of CLDN5-overexpressing and control hCMEC/D3 cells were extracted using TRIzol Reagent (Invitrogen Life Technologies, Carlsbad, CA, USA) according to the manufacturer's protocol. RNA quantity was evaluated by the $28 \mathrm{~S} / 18 \mathrm{~S}$ ratio and RNA Integrity Number (RIN) was analyzed on the Agilent 2100 bioanalyzer using the Eukaryote Total RNA Nano assay (Agilent Technologies, Waldbronn, Germany). RNA purity was evaluated by the A260/A280 ratio using an RNA 6000 Nano Spectrophotometer (Agilent).

miRNA, mRNA, lncRNA microarray and computational analysis. For mRNA and lncRNA expression profiling, Affymetrix Human Transcriptome Array 2.0 (HTA 2.0) (Thermo Fisher Scientific, Inc.) was used. For miRNA expression profiling, Affymetrix GeneChip miRNA 4.0 Array (Thermo Fisher Scientific, Inc.) was used. After hybridization and washing, the arrays were scanned by an Affymetrix Microarray Scanner (Applied Biosystems, Grand Island, NY, USA). Raw data of HTA 2.0 were extracted and normalized by Affymetrix ${ }^{\circledR}$ Transcriptome Analysis Console (TAC) Software (Thermo Fisher Scientific, Inc.). miRNA QC Tool software (Thermo Fisher Scientific, Inc.) was used for miRNA 4.0 array data summarization, normalization, and quality control.

Difference analysis. Two-class differential was used to determine the differentially expressed miRNA, lncRNA and mRNA between the pLL3.7-CLDN5-transfected group $(n=3)$ and pLL3.7 control group $(n=3)$. The random variance model (RVM) t-test was applied to filter the differentially expressed genes for it can effectively raise the degrees of freedom in cases of small samples. The false discovery rate (FDR) was calculated to correct the P-value. P-values $<0.05$ and FDR $<0.05$ were considered as significant differences.

The differentially expressed probe sets were imported into Cluster and Tree View to perform hierarchical cluster analysis (HCA).

Construction of lncRNA-mRNA co-expression network. We utilized the expression profile of different lncRNAs and difference mRNAs to construct the IncRNA-mRNA network. This network distinctly revealed the relation between the lncRNAs and mRNAs, found the key regulation and 'interaction venation' thoroughly. This network assimilated the scale-free property of the huge data, to simulate the scale-free relation by the interaction between IncRNAs and mRNAs and interaction among themselves, and that is the correlation of pairwise expression. In order to get reliable and accurate relation of gene interaction and IncRNA regulation function, we recommend the number of no less than 30 and the number of genes no less than 200 .

For each pair of mRNA-lncRNA, mRNA-mRNA, or lncRNA-lncRNA, we calculated the Pearson correlation 
Table I. The primers of miRNA, mRNA, and lncRNA for qRT-PCR.

\begin{tabular}{|c|c|}
\hline Primers & Sequence $\left(5^{\prime}-3^{\prime}\right)$ \\
\hline miR-297 & $\begin{array}{l}\text { RT: TCAACTGGTGTCGTGGAGTCGGCAATTCAGTTGACATGCACA } \\
\text { F: CTCAACTGGTGTCGTGGAGT } \\
\text { R: ACACTCCAGCTGGGATGTATGTGTGCAT }\end{array}$ \\
\hline miR-610 & $\begin{array}{l}\text { RT: CTCAACTGGTGTCGTGGAGTCGGCAATTCAGTTGATCCCAGCA } \\
\text { F: CTCAACTGGTGTCGTGGAGT } \\
\text { R: ACACTCCAGCTGGGTGAGCTAAATGTG }\end{array}$ \\
\hline $\operatorname{miR}-767-5 p$ & $\begin{array}{l}\text { RT: CTCAACTGGTGTCGTGGAGTCGGCAATTCAGTTGACATGCTCA } \\
\text { F: CTCAACTGGTGTCGTGGAGT } \\
\text { R: ACACTCCAGCTGGGTGCACCATGGTTGTCT }\end{array}$ \\
\hline miR-329-3P & $\begin{array}{l}\text { RT:CTCAACTGGTGTCGTGGAGTCGGCAATTCAGTTGAAAAGAGGT } \\
\text { F: CTCAACTGGTGTCGTGGAGT } \\
\text { R: ACACTCCAGCTGGGAACACACCTGGTTAA }\end{array}$ \\
\hline miR-921 & $\begin{array}{l}\text { RT: CTCAACTGGTGTCGTGGAGTCGGCAATTCAGTTGAGAATCCTG } \\
\text { F: CTCAACTGGTGTCGTGGAGT } \\
\text { R: ACACTCCAGCTGGGCTAGTGAGGGACAGAACC }\end{array}$ \\
\hline $\operatorname{miR}-127-5 p$ & $\begin{array}{l}\text { RT: CTCAACTGGTGTCGTGGAGTCGGCAATTCAGTTGAATCAGAGC } \\
\text { F: CTCAACTGGTGTCGTGGAGT } \\
\text { R: ACACTCCAGCTGGGCTGAAGCTCAGAGGG }\end{array}$ \\
\hline $\operatorname{miR}-4786-5 p$ & $\begin{array}{l}\text { RT: CTCAACTGGTGTCGTGGAGTCGGCAATTCAGTTGAGGTGCATC } \\
\text { F: CTCAACTGGTGTCGTGGAGT } \\
\text { R: ACACTCCAGCTGGGTGAGACCAGGACTGG }\end{array}$ \\
\hline POTED & $\begin{array}{l}\text { F: GTGGTGCTGTCACTGCTTCCC } \\
\text { R: CGCTGTGGTCGTAGTCTCCC }\end{array}$ \\
\hline TMPRSS15 & $\begin{array}{l}\text { F: GGCAGCTCAAGACATCACCC } \\
\text { R: GCCGCCATAATACAGACCCA }\end{array}$ \\
\hline EFNB2 & $\begin{array}{l}\text { F: GAGCAGGAAGCCGATGTGAC } \\
\text { R: GGGAAAACCCAACGCAGAAA }\end{array}$ \\
\hline IL7R & $\begin{array}{l}\text { F: TGAGTGTCGTCTATCGGGAAGG } \\
\text { R: CTGGCGGTAAGCTACATCGTG }\end{array}$ \\
\hline AMTN & $\begin{array}{l}\text { F: CAGACCCACCCATTGACCCT } \\
\text { R: GGATTAGCCCCTGCCTGACT }\end{array}$ \\
\hline PIK3CG & $\begin{array}{l}\text { F: GCCACTGATCCACTTAACCCTC } \\
\text { R: AATTTCTTGCTGTCCCCATTTC }\end{array}$ \\
\hline CLDN1 & $\begin{array}{l}\text { F: TGAGGATGGCTGTCATTGGG } \\
\text { R: ACCTGGCATTGACTGGGGTC }\end{array}$ \\
\hline OCLN & $\begin{array}{l}\text { F: TCTCСCTCCCTGCTTCCTCT } \\
\text { R: GCAATGCCCTTTAGCTTCCA }\end{array}$ \\
\hline ENST00000427446 & $\begin{array}{l}\text { F: ATTATCAGCTCGTGAGTACGGACAT } \\
\text { R: GAAAACATGGAAGCTCTATTTGGTC }\end{array}$ \\
\hline n342142 & $\begin{array}{l}\text { F: AGAAAGAGCCAACAACTCCTACAGA } \\
\text { R: TGAGTGGTTCAGATTTAGGCACAGA }\end{array}$ \\
\hline $\begin{array}{l}\text { TCONS_00017956- } \\
\text { XLOC_008786 }\end{array}$ & $\begin{array}{l}\text { F: GATAATTCCGTTTGACTCCGTTTGA } \\
\text { R: CTAATGGAATCGCATGGTATCTTCA }\end{array}$ \\
\hline ENST00000425979 & $\begin{array}{l}\text { F: CAAAGGCAAAGATTGGAGTTGTACT } \\
\text { R: ACGAATCCTTGCTTGTTTCTTCTAG }\end{array}$ \\
\hline $\begin{array}{l}\text { TCONS_00028418- } \\
\text { XLOC_013771 }\end{array}$ & $\begin{array}{l}\text { F: CTTTCTGGGTCTTCTGTTTCACGAT } \\
\text { R: TTTCTGTTCAAGTTTGAGGGTCCTG }\end{array}$ \\
\hline n338895 & $\begin{array}{l}\text { F: GCACGGTATCATTACTCCCGTTTTA } \\
\text { R: AACCTCTGATAAGGACAAGCAACCC }\end{array}$ \\
\hline
\end{tabular}


Table I. Continued.

Primers

Sequence (5'-3')

n339695

F: ATTCTCCTGCCTCAGCCTCCCAAGT

TCONS_00022673-XLOC_010971

R: GTGCGGTGGCTCACGTCTGTAGTCC

F: AACAGAAGCCCAGGGAGATAAAGAC

R: TGGAAATGATCCTGGATTAGGAATG

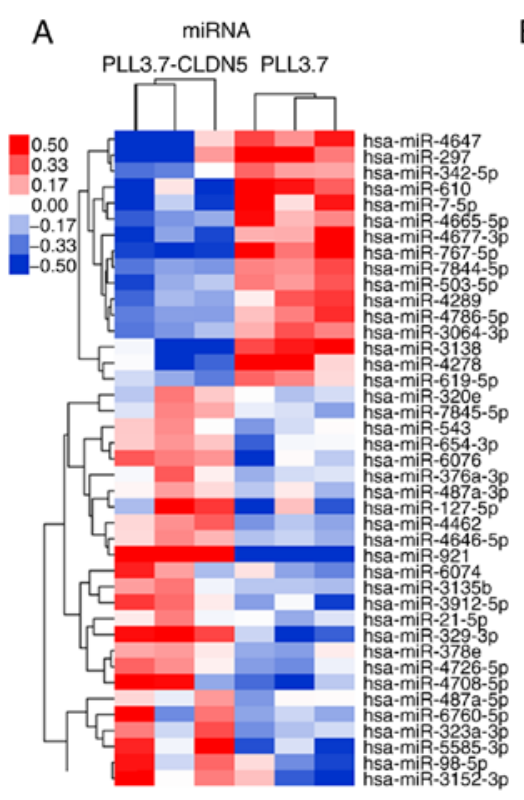

B $\quad$ mRNA

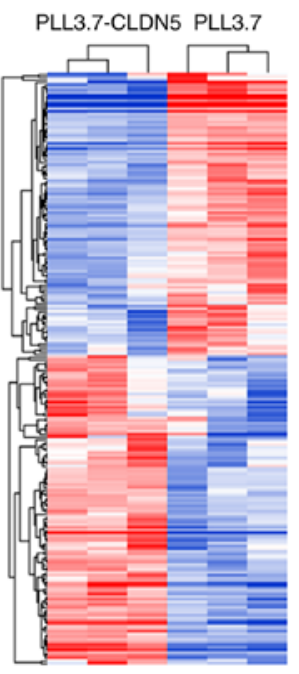

C LICRNA PLL3.7-CLDN5 PLL3.7

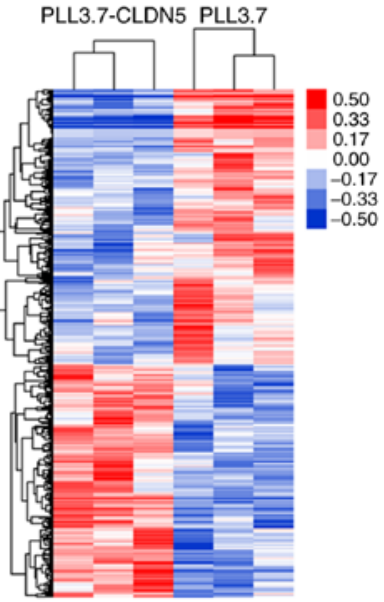

Figure 1. Hierarchical clustering analysis of miRNA, LncRNA, and mRNA expression levels between pLL3.7-CLDN5-transfected group ( $\mathrm{n}=3$ ) and pLL3.7 control group (n=3). (A) 41 miRNAs, (B) 954 lncRNAs and (C) 222 mRNAs are differentially expressed in overexpressing hCMEC/D3 cells, fold change $\geq 1.2$ or $\leq 0.8, \mathrm{P}<0.05$. Columns represent samples and rows respectively represent miRNA, IncRNA or mRNA probe sets. Red represents high expression, blue represents low expression, indicating expression above and below the median expression value across all the samples (log scale 2: From -0.5 to +0.5$)$.

and chose the significant correlation pairs to construct the network (20). The clustering coefficient represents the density of each gene with the adjacent gene, and the larger the clustering coefficient, the greater importance the gene has in regulating the network.

Gene ontology and pathway analysis. A gene ontology (GO) analysis was applied to analyze the main functions of the mRNAs of miRNA-lncRNA-mRNA internets. Specifically, a two-side Fisher's exact test and a $\chi^{2}$ test were used to classify the GO category. We computed P-values of the GO for each differential gene. Enrichment provides a measure for the significant function: As the enrichment increases, the corresponding function is more specific. Within the significant category, the enrichment Re was given as follows: $\operatorname{Re}=\left(n_{f} / n\right) /\left(N_{f} / N\right)$, where $n_{f}$ is the number of flagged genes within the particular category, $n$ is the total number of genes within the same category, $N_{f}$ is the number of flagged genes in the entire microarray, and $N$ is the total number of genes in the microarray.

Pathway analysis was used to identify the significant pathway of the differential mRNAs according to KEGG, Biocarta, and Reatome. We used Fisher's exact test and the $\chi^{2}$ test to select the significant pathway, and the threshold of

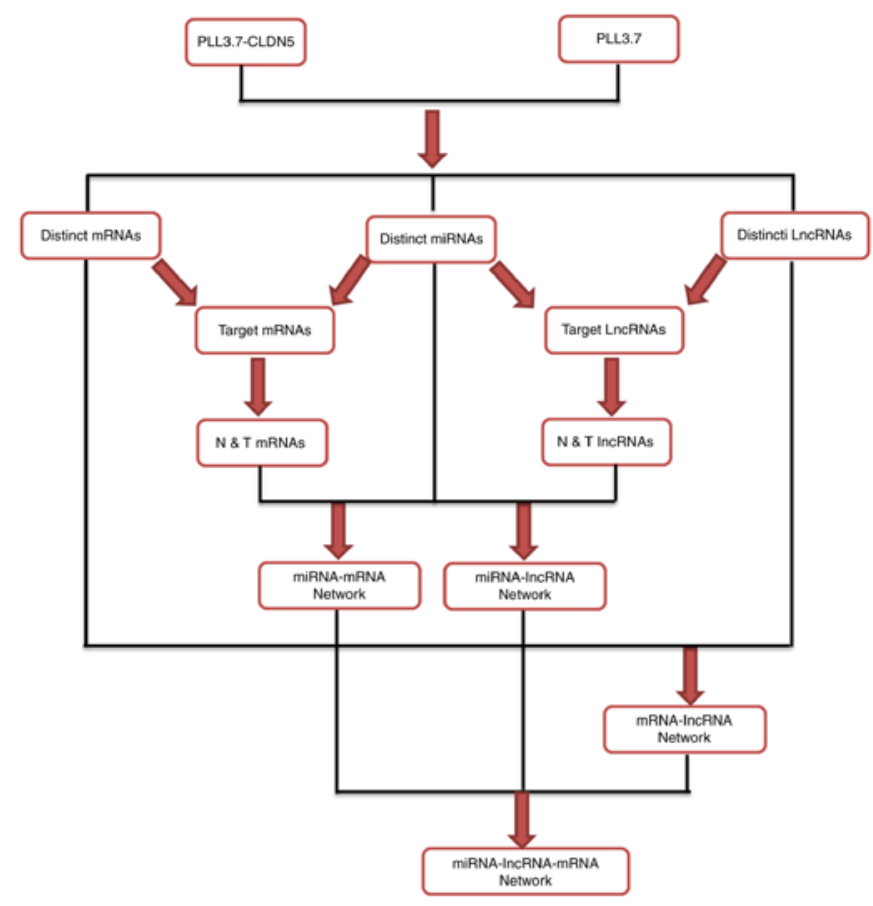

Figure 2. Schematic overview of the work flow for construction of miRNA-lncRNA-mRNA interaction network. 
Table II. 146 lncRNAs as ceRNAs in the miRNA-lncRNA-mRNA interaction network.

\begin{tabular}{|c|c|c|c|}
\hline No. & lncRNA accession & No. & lncRNA accession \\
\hline 1 & TCONS_12_00014900-XLOC_12_008262 & 75 & n334798 \\
\hline 2 & TCONS_12_00020697-XLOC_12_010802 & 76 & n338832 \\
\hline 3 & TCONS_00016931-XLOC_007962 & 77 & n340212 \\
\hline 4 & TCONS_12_00016969-XLOC_12_008979 & 78 & n340287 \\
\hline 5 & TCONS_00008436-XLOC_003882 & 79 & n382215 \\
\hline 6 & TCONS_12_00016970-XLOC_12_008980 & 80 & n405896 \\
\hline 7 & TCONS_12_00017319-XLOC_12_008977 & 81 & n410552 \\
\hline 8 & NR_002813 & 82 & NR_027755 \\
\hline 9 & TCONS_12_00012202-XLOC_12_006419 & 83 & TCONS_00012035-XLOC_005569 \\
\hline 10 & n409500 & 84 & TCONS_00014701-XLOC_006799 \\
\hline 11 & n341154 & 85 & TCONS_00017991-XLOC_008840 \\
\hline 12 & TCONS_12_00010766-XLOC_12_005781 & 86 & TCONS_00024504-XLOC_011823 \\
\hline 13 & n342063 & 87 & ENST00000448869 \\
\hline 14 & n386722 & 88 & ENST00000506895 \\
\hline 15 & TCONS_00003006-XLOC_001678 & 89 & n326341 \\
\hline 16 & NR_024387 & 90 & n332799 \\
\hline 17 & TCONS_00007677-XLOC_003770 & 91 & n335107 \\
\hline 18 & TCONS_00019368-XLOC_009191 & 92 & n335724 \\
\hline 19 & n409372 & 93 & n337920 \\
\hline 20 & n342249 & 94 & n338102 \\
\hline 21 & n408882 & 95 & n338270 \\
\hline 22 & TCONS_00022673-XLOC_010971 & 96 & n340792 \\
\hline 23 & n334377 & 97 & n378134 \\
\hline 24 & NR_024076 & 98 & n382996 \\
\hline 25 & NR_033360 & 99 & n406201 \\
\hline 26 & NR_038399 & 100 & n407038 \\
\hline 27 & TCONS_00016279-XLOC_007654 & 101 & n408084 \\
\hline 28 & TCONS_00026192-XLOC_012669 & 102 & n409198 \\
\hline 29 & TCONS_00028418-XLOC_013771 & 103 & NR_036676 \\
\hline 30 & ENST00000440955 & 104 & OTTHUMT00000318709 \\
\hline 31 & n341106 & 105 & TCONS_00009631-XLOC_004772 \\
\hline 32 & n409178 & 106 & TCONS_00012197-XLOC_005754 \\
\hline 33 & NR_027995 & 107 & TCONS_00026389-XLOC_012738 \\
\hline 34 & TCONS_00011934-XLOC_005442 & 108 & TCONS_12_00020780-XLOC_12_010854 \\
\hline 35 & TCONS_00028427-XLOC_013779 & 109 & TCONS_12_00025904-XLOC_12_013423 \\
\hline 36 & TCONS_00011225-XLOC_005777 & 110 & ENST00000422082 \\
\hline 37 & TCONS_00028805-XLOC_013878 & 111 & ENST00000468202 \\
\hline 38 & n338895 & 112 & ENST00000511994 \\
\hline 39 & n339264 & 113 & ENST00000513211 \\
\hline 40 & n381942 & 114 & ENST00000514265 \\
\hline 41 & NR_002808 & 115 & ENST00000546710 \\
\hline 42 & TCONS_00020860-XLOC_010143 & 116 & ENST00000550035 \\
\hline 43 & TCONS_00024807-XLOC_011645 & 117 & n324907 \\
\hline 44 & TCONS_12_00011136-XLOC_12_006021 & 118 & n325126 \\
\hline 45 & n338919 & 119 & n325417 \\
\hline 46 & n339042 & 120 & n325964 \\
\hline 47 & n341945 & 121 & n332690 \\
\hline 48 & n342142 & 122 & n333177 \\
\hline 49 & n411744 & 123 & n333293 \\
\hline 50 & NR_038849 & 124 & n335571 \\
\hline 51 & TCONS_00009713-XLOC_004905 & 125 & n339266 \\
\hline
\end{tabular}


Table II. Continued.

\begin{tabular}{|c|c|c|c|}
\hline No. & lncRNA accession & No. & lncRNA accession \\
\hline 52 & ENST00000414772 & 126 & n339695 \\
\hline 53 & ENST00000427446 & 127 & n340730 \\
\hline 54 & ENST00000508925 & 128 & n340955 \\
\hline 55 & ENST00000546560 & 129 & n342367 \\
\hline 56 & n340540 & 130 & n342493 \\
\hline 57 & n342109 & 131 & n345192 \\
\hline 58 & n379390 & 132 & n345550 \\
\hline 59 & n411650 & 133 & n346330 \\
\hline 60 & TCONS_00028187-XLOC_013543 & 134 & n346394 \\
\hline 61 & TCONS_12_00021562-XLOC_12_010625 & 135 & n383730 \\
\hline 62 & n342193 & 136 & n408256 \\
\hline 63 & n342616 & 137 & $\mathrm{n} 408302$ \\
\hline 64 & n342622 & 138 & n410892 \\
\hline 65 & n406194 & 139 & n411520 \\
\hline 66 & n406899 & 140 & NR_033752 \\
\hline 67 & n411603 & 141 & NR_033826 \\
\hline 68 & OTTHUMT00000363702 & 142 & OTTHUMT00000370627 \\
\hline 69 & TCONS_00016056-XLOC_007446 & 143 & TCONS_00000861-XLOC_000086 \\
\hline 70 & TCONS_00022099-XLOC_010709 & 144 & TCONS_00003277-XLOC_002089 \\
\hline 71 & TCONS_12_00012572-XLOC_12_006751 & 145 & TCONS_00008003-XLOC_003469 \\
\hline 72 & ENST00000452532 & 146 & TCONS_00017956-XLOC_008786 \\
\hline 73 & ENST00000507838 & 147 & TCONS_00025335-XLOC_012147 \\
\hline 74 & n325350 & 148 & TCONS_00029068-XLOC_013994 \\
\hline
\end{tabular}

significance was defined by P-value and FDR. The enrichment Re was calculated as indicated in the equation above.

$q R T-P C R$ analysis. Total RNA was reverse-transcribed with a PrimeScript ${ }^{\mathrm{TM}}$ RT reagent kit (Takara Biotechnology Co., Ltd., Dalian, China) for cDNA synthesis and genomic DNA removal. For miRNA detection, total RNA was reverse transcribed using miRNA specific primers. QPCR was performed according to the instructions of the SYBR premix Ex Taq ${ }^{\mathrm{TM}}$ II kit (Takara Biotechnology Co.) and carried out in the Takara real-time PCR system. GAPDH was used as lncRNA and mRNA control and U6 was used as a miRNA control. Gene-specific primers were designed using primer designing tools primer 5.0. The primer sequences are listed in Table I. The specificity of amplification was assessed by dissociation curve analysis, and the relative abundance of genes was determined with the $2^{-\Delta \Delta \mathrm{Ct}}$ method. All experiments were performed in triplicate.

\section{Results}

CLDN-5 induces significantly altered miRNA, IncRNA and $m R N A$ expression patterns in $h C M E C / D 3$ cells. To explore the regulatory mechanism of CLDN5 on permeability of BBB, we stably transfected the hCMEC/D3 cells with pLL3.7-CLDN5 or pLL3.7, and then analyzed the changes of miRNA, IncRNA, and mRNA levels in CLDN-5-overexpressing hCMEC/D3 cells.

In terms of the miRBaseV20 Database, 2578 human miRNAs were authenticated on the Affymetrix GeneChip
microRNA 4.0 Array. Based on the RefSeq, UCSC, GENCODES, MGC, lincRNAs TUCPs, lincRNAdb and UCSC lincRNA, annotations of lncRNAs and mRNAs, the probe sets covered 22, 829 lncRNAs and 44, 699 mRNAs on the Affymetrix GeneChip HTA 2.0 Array.

The miRNA, lncRNA, and mRNA expression patterns were detected in pLL3.7-CLDN5-transfected group and pLL3.7 control group. We identified 41 miRNAs, 954 lncRNAs, and 222 mRNAs that had significant differential expression in the CLDN5-overexpressing group comparing with the control group (fold change $\geq 1.2$ or $\leq 0.8$, and P-value $<0.05$ ). The hierarchical clustering analysis showed that with the differentially expression of these miRNAs, lncRNAs and mRNAs, samples were non-random partitioned, they were divided into two groups (Fig. 1). Thus, the miRNA, lncRNA, and mRNA expression signatures identified here were likely to be representative.

Construction of miRNA-lncRNA-mRNA interaction of network regulated by reinforced CLDN5 and identification lncRNAs acting as ceRNAs. The miRNA-lncRNA-mRNA interaction network was constructed according to the work flow summarized in Fig. 2.

Firstly, the miRanda was applied to analyze the target mRNAs and lncRNAs of the 41 miRNAs, and then the interaction of these target mRNAs with distinct mRNAs and target lncRNAs with distinct lncRNAs, separately termed as target mRNAs (172 mRNAs, data not shown) and target lncRNAs 
Table III. The 95 feed-forward loops including miRNAs, lncRNAs and mRNAs.

\begin{tabular}{|c|c|c|c|}
\hline No. & miRNA & lncRNA & mRNA \\
\hline 1 & hsa-miR-4677-3p & n325350 & IGFBP3 \\
\hline 2 & hsa-miR-487a-3p & TCONS_00012035-XLOC_005569 & PIK3CG \\
\hline 3 & hsa-miR-6760-5p & TCONS_00012035-XLOC_005569 & PIK3CG \\
\hline 4 & hsa-miR-3135b & NR_038399 & DOCK1 \\
\hline 5 & hsa-miR-6760-5p & NR_038399 & DOCK1 \\
\hline 6 & hsa-miR-297 & NR_033360 & IGFBP3 \\
\hline 7 & hsa-miR-4677-3p & NR_033360 & IGFBP3 \\
\hline 8 & hsa-miR-297 & TCONS_00009713-XLOC_004905 & IGFBP3 \\
\hline 9 & hsa-miR-4289 & TCONS_00009713-XLOC_004905 & IGFBP3 \\
\hline 10 & hsa-miR-619-5p & TCONS_00009713-XLOC_004905 & IGFBP3 \\
\hline 11 & hsa-miR-7-5p & TCONS_00009713-XLOC_004905 & IGFBP3 \\
\hline 12 & hsa-miR-4726-5p & TCONS_12_00011136-XLOC_12_006021 & DOCK1 \\
\hline 13 & hsa-miR-6760-5p & TCONS_12_00011136-XLOC_12_006021 & DOCK1 \\
\hline 14 & hsa-miR-323a-3p & TCONS_00003006-XLOC_001678 & CDKN1B \\
\hline 15 & hsa-miR-3912-5p & TCONS_00003006-XLOC_001678 & CDKN1B \\
\hline 16 & hsa-miR-543 & TCONS_00003006-XLOC_001678 & CDKN1B \\
\hline 17 & hsa-miR-6760-5p & TCONS_00003006-XLOC_001678 & CDKN1B \\
\hline 18 & hsa-miR-7845-5p & TCONS_00003006-XLOC_001678 & CDKN1B \\
\hline 19 & hsa-miR-3912-5p & TCONS_00003006-XLOC_001678 & PHLPP1 \\
\hline 20 & hsa-miR-6760-5p & TCONS_00003006-XLOC_001678 & PHLPP1 \\
\hline 21 & hsa-miR-7845-5p & TCONS_00003006-XLOC_001678 & PHLPP1 \\
\hline 22 & hsa-miR-297 & TCONS_12_00021562-XLOC_12_010625 & IGFBP3 \\
\hline 23 & hsa-miR-4289 & TCONS_12_00021562-XLOC_12_010625 & IGFBP3 \\
\hline 24 & hsa-miR-4677-3p & TCONS_12_00021562-XLOC_12_010625 & IGFBP3 \\
\hline 25 & hsa-miR-619-5p & TCONS_12_00021562-XLOC_12_010625 & IGFBP3 \\
\hline 26 & hsa-miR-7-5p & TCONS_12_00021562-XLOC_12_010625 & IGFBP3 \\
\hline 27 & hsa-miR-297 & n409198 & IGFBP3 \\
\hline 28 & hsa-miR-4677-3p & n409198 & IGFBP3 \\
\hline 29 & hsa-miR-3135b & n406899 & ARHGEF12 \\
\hline 30 & hsa-miR-4462 & n406899 & ARHGEF12 \\
\hline 31 & hsa-miR-654-3p & n406899 & ARHGEF12 \\
\hline 32 & hsa-miR-7845-5p & n406899 & ARHGEF12 \\
\hline 33 & hsa-miR-3135b & TCONS_12_00014900-XLOC_12_008262 & DOCK1 \\
\hline 34 & hsa-miR-323a-3p & TCONS_12_00014900-XLOC_12_008262 & DOCK1 \\
\hline 35 & hsa-miR-329-3p & TCONS_12_00014900-XLOC_12_008262 & DOCK1 \\
\hline 36 & hsa-miR-4462 & TCONS_12_00014900-XLOC_12_008262 & DOCK1 \\
\hline 37 & hsa-miR-4726-5p & TCONS_12_00014900-XLOC_12_008262 & DOCK1 \\
\hline 38 & hsa-miR-6076 & TCONS_12_00014900-XLOC_12_008262 & DOCK1 \\
\hline 39 & hsa-miR-6760-5p & TCONS_12_00014900-XLOC_12_008262 & DOCK1 \\
\hline 40 & hsa-miR-921 & TCONS_12_00014900-XLOC_12_008262 & DOCK1 \\
\hline 41 & hsa-miR-6760-5p & n346394 & CADM1 \\
\hline 42 & hsa-miR-4665-5p & ENST00000546560 & PPARD \\
\hline 43 & hsa-miR-323a-3p & TCONS_12_00020697-XLOC_12_010802 & CDKN1B \\
\hline 44 & hsa-miR-3912-5p & TCONS_12_00020697-XLOC_12_010802 & CDKN1B \\
\hline 45 & hsa-miR-543 & TCONS_12_00020697-XLOC_12_010802 & CDKN1B \\
\hline 46 & hsa-miR-6760-5p & TCONS_12_00020697-XLOC_12_010802 & CDKN1B \\
\hline 47 & hsa-miR-7845-5p & TCONS_12_00020697-XLOC_12_010802 & CDKN1B \\
\hline 48 & hsa-miR-4289 & n335571 & IGFBP3 \\
\hline 49 & hsa-miR-4278 & ENST00000452532 & PPARD \\
\hline 50 & hsa-miR-3912-5p & TCONS_12_00020697-XLOC_12_010802 & PHLPP1 \\
\hline 51 & hsa-miR-4646-5p & TCONS_12_00020697-XLOC_12_010802 & PHLPP1 \\
\hline 52 & hsa-miR-6760-5p & TCONS_12_00020697-XLOC_12_010802 & PHLPP1 \\
\hline
\end{tabular}


Table III. Continued.

\begin{tabular}{|c|c|c|c|}
\hline No. & miRNA & lncRNA & mRNA \\
\hline 53 & hsa-miR-7845-5p & TCONS_12_00020697-XLOC_12_010802 & PHLPP1 \\
\hline 54 & hsa-miR-4289 & n333293 & CDKN1A \\
\hline 55 & hsa-miR-3135b & n409500 & DOCK1 \\
\hline 56 & hsa-miR-4462 & n409500 & DOCK1 \\
\hline 57 & hsa-miR-4726-5p & n409500 & DOCK1 \\
\hline 58 & hsa-miR-6076 & n409500 & DOCK1 \\
\hline 59 & hsa-miR-6760-5p & n409500 & DOCK1 \\
\hline 60 & hsa-miR-297 & n408084 & OCLN \\
\hline 61 & hsa-miR-4647 & n408084 & OCLN \\
\hline 62 & hsa-miR-3135b & TCONS_12_00020697-XLOC_12_010802 & DOCK1 \\
\hline 63 & hsa-miR-323a-3p & TCONS_12_00020697-XLOC_12_010802 & DOCK1 \\
\hline 64 & hsa-miR-4462 & TCONS_12_00020697-XLOC_12_010802 & DOCK1 \\
\hline 65 & hsa-miR-4726-5p & TCONS_12_00020697-XLOC_12_010802 & DOCK1 \\
\hline 66 & hsa-miR-6760-5p & TCONS_12_00020697-XLOC_12_010802 & DOCK1 \\
\hline 67 & hsa-miR-921 & TCONS_12_00020697-XLOC_12_010802 & DOCK1 \\
\hline 68 & hsa-miR-619-5p & TCONS_00029068-XLOC_013994 & CDKN1A \\
\hline 69 & hsa-miR-3135b & NR_024387 & ARHGEF12 \\
\hline 70 & hsa-miR-4462 & NR_024387 & ARHGEF12 \\
\hline 71 & hsa-miR-654-3p & NR_024387 & ARHGEF12 \\
\hline 72 & hsa-miR-7845-5p & NR_024387 & ARHGEF12 \\
\hline 73 & hsa-miR-3912-5p & TCONS_00008436-XLOC_003882 & PHLPP1 \\
\hline 74 & hsa-miR-4646-5p & TCONS_00008436-XLOC_003882 & PHLPP1 \\
\hline 75 & hsa-miR-6760-5p & TCONS_00008436-XLOC_003882 & PHLPP1 \\
\hline 76 & hsa-miR-7845-5p & TCONS_00008436-XLOC_003882 & PHLPP1 \\
\hline 77 & hsa-miR-297 & ENST00000452532 & OCLN \\
\hline 78 & hsa-miR-4786-5p & n339695 & CLDN1 \\
\hline 79 & hsa-miR-4726-5p & n332799 & DOCK1 \\
\hline 80 & hsa-miR-127-5p & n338895 & PIK3CG \\
\hline 81 & hsa-miR-378e & n338895 & PIK3CG \\
\hline 82 & hsa-miR-5585-3p & n338895 & PIK3CG \\
\hline 83 & hsa-miR-6760-5p & n338895 & PIK3CG \\
\hline 84 & hsa-miR-7845-5p & n338895 & PIK3CG \\
\hline 85 & hsa-miR-297 & TCONS_00022673-XLOC_010971 & OCLN \\
\hline 86 & hsa-miR-3138 & TCONS_00022673-XLOC_010971 & OCLN \\
\hline 87 & hsa-miR-4647 & TCONS_00022673-XLOC_010971 & OCLN \\
\hline 88 & hsa-miR-4786-5p & TCONS_00022673-XLOC_010971 & OCLN \\
\hline 89 & hsa-miR-619-5p & TCONS_00022673-XLOC_010971 & OCLN \\
\hline 90 & hsa-miR-7-5p & TCONS_00022673-XLOC_010971 & OCLN \\
\hline 91 & hsa-miR-767-5p & TCONS_00022673-XLOC_010971 & OCLN \\
\hline 92 & hsa-miR-7844-5p & TCONS_00022673-XLOC_010971 & OCLN \\
\hline 93 & hsa-miR-3138 & TCONS_00024504-XLOC_011823 & CDKN1A \\
\hline 94 & hsa-miR-4677-3p & TCONS_00024504-XLOC_011823 & CDKN1A \\
\hline 95 & hsa-miR-619-5p & TCONS_00024504-XLOC_011823 & CDKN1A \\
\hline
\end{tabular}

(681 lncRNAs, data not shown). Of the target mRNAs and IncRNAs, the mRNAs and IncRNAs were selected which expression levels were negatively correlated with miRNA expression, and were termed the N\&T mRNAs (152 mRNAs, data not shown) and N\&T IncRNAs (618 lncRNAs, data not shown), and obtained the miRNA-mRNA, miRNA-IncRNA network.
Secondly, according to the correlation of gene expression of 222 mRNAs and 954 lncRNAs, we constructed the lncRNA-mRNA co-expression network (data not shown).

At last, based on the interaction network of miRNAmRNA, miRNA-lncRNA, and IncRNA-mRNA, we obtained 1049 feed-forward loop networks and constructed the general 
A
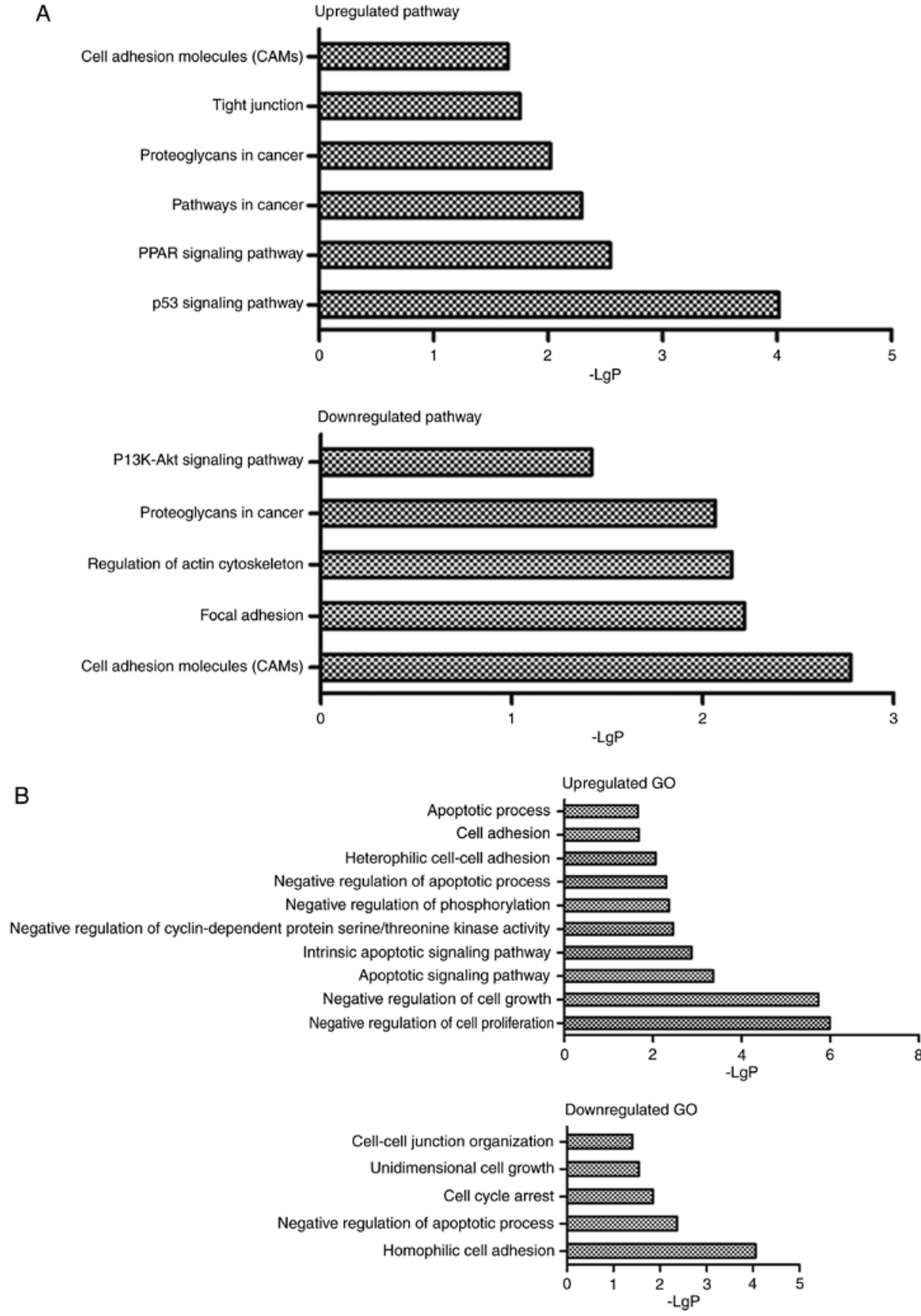

Figure 3. Function prediction of the ceRNAs based on pathway and GO analysis of mRNAs located in miRNA-lncRNA-mRNA interaction network. (A) The pathway analysis showed the 6 upregulated and 5 downregulated signaling pathways $(\mathrm{P}<0.05)$, and (B) GO analysis showed the 10 upregulated and 5 downregulated functions $(\mathrm{P}<0.05)$.

miRNA-lncRNA-mRNA interaction network (data not shown). In this network, we got 148 miRNAs targeted lncRNAs, and these 148 lncRNAs were identified as ceRNAs and listed in Table II.

Biological role prediction of IncRNAs function as ceRNAs in CLDN5-overexpressing hCMEC/D3 cells. GO and pathway analysis were applied to analyze the significant function and pathway of the mRNAs that contained in the 1049 feed-forward loop networks. Go analysis results showed that upregulated and downregulated mRNA respectively, were involved in 122 and 81 items significant functions (P-value $<0.05$, data not shown). The pathway analysis revealed that there were 29 and 6 significant pathways corresponding to the up and down regulated mRNAs separately (P-value $<0.05$, data not shown).

Further analysis indicated there are 10 mRNAs that contained both in the significant function and pathway (Table III), and they participated in 95 feed-forward loop networks. The miRNAs, lncRNAs, and mRNAs in 95 feed-forward loop are listed in Table IV.

The biological roles of 27 lncRNAs acting as ceRNAs were predicted through GO and pathway analysis of 10 mRNAs in the miRNA-lncRNA-mRNA interaction network. In addition, the results indicated that 10 mRNAs participated in 6 upregulated and 5 downregulated pathways involving in diverse biological processes, including tight junction, focal 
Table IV. Functional prediction of the IncRNA ceRNAs based on pathway and GO analysis of mRNAs that locate together in the miRNA-lncRNA-mRNA feed-forward loop.

\begin{tabular}{|c|c|c|c|}
\hline No. & mRNA & Pathway & $\mathrm{GO}$ \\
\hline 1 & IGFBP3 & P53 signaling & $\begin{array}{l}\text { Negative regulation of cell proliferation } \\
\text { Regulation of cell growth } \\
\text { Negative regulation of protein phosphorylation } \\
\text { Apoptotic process }\end{array}$ \\
\hline 2 & PIK3CG & $\begin{array}{l}\text { Focal adhesion } \\
\text { Regulation of actin cytoskeleton } \\
\text { Proteoglycans in cancer } \\
\text { PI3K-Akt signaling }\end{array}$ & Negative regulation of apoptotic process \\
\hline 3 & CDKN1A & $\begin{array}{l}\text { P53 signaling } \\
\text { Pathways in cancer } \\
\text { Proteoglycans in cancer }\end{array}$ & $\begin{array}{l}\text { Negative regulation of cell proliferation/cell growth } \\
\text { Intrinsic apoptotic signaling } \\
\text { Negative regulation of cyclin-dependent protein } \\
\text { serine/threonine kinase activity } \\
\text { Negative regulation of phosphorylation } \\
\text { Negative regulation of apoptotic process }\end{array}$ \\
\hline 4 & CDKN1B & PI3K-Akt signaling & $\begin{array}{l}\text { Negative regulation of apoptotic process } \\
\text { Cell cycle arrest }\end{array}$ \\
\hline 5 & PHLPP1 & PI3K-Akt signaling & Apoptotic process \\
\hline 6 & ARHGEF12 & $\begin{array}{l}\text { Regulation of actin cytoskeleton } \\
\text { Proteoglycans in cancer }\end{array}$ & \\
\hline 7 & PPARD & $\begin{array}{l}\text { PPAR signaling pathway } \\
\text { Pathways in cancer }\end{array}$ & Apoptotic signaling \\
\hline 8 & CADM1 & Cell adhesion molecules & $\begin{array}{l}\text { Homophilic cell adhesion } \\
\text { Apoptotic process } \\
\text { Heterophilic cell-cell adhesion } \\
\text { Unidimensional cell growth } \\
\text { Cell-cell junction organization }\end{array}$ \\
\hline 9 & CLDN1 & $\begin{array}{l}\text { Tight junction } \\
\text { Cell adhesion molecules }\end{array}$ & $\begin{array}{l}\text { Cell adhesion } \\
\text { Cell-cell junction organization }\end{array}$ \\
\hline 10 & DOCK1 & $\begin{array}{l}\text { Focal adhesion; } \\
\text { Regulation of actin cytoskeleton }\end{array}$ & Apoptotic process \\
\hline 11 & OCLN & $\begin{array}{l}\text { Apoptotic process; } \\
\text { Cell-cell junction organization }\end{array}$ & $\begin{array}{l}\text { Tight junction } \\
\text { Cell adhesion molecules }\end{array}$ \\
\hline
\end{tabular}

adhesion and cell adhesion molecules, and 10 upregulated and 5 downregulated functions that containing cell adhesion, heterophilic cell-cell adhesion, cell growth, cell apoptosis, etc (Fig. 3).

As a consequence, we predicted the important roles of the 27 ceRNAs in CLDN-5-overexpressing hCMEC/D3 cells.

Quantitative real-time $R T-P C R$ ( $q R T-P C R)$ analysis of the distinctive expression of miRNAs, lncRNAs, and mRNAs in hCMEC/D3 cells with overexpressed CLDN5. To validate the results of microarray analysis, we selected 5 miRNAs, 5 mRNAs, and 5 lncRNAs with larger foldchange from the microarray results and analyzed their expression levels by qRT-PCR in pLL3.7-CLDN5 and pLL3.7 hCMEC/D3 cells. Our results confirmed the findings of the miRNAs, lncRNAs and mRNAs microarray dataset (Fig. 4A).

Based on the analysis of 95 miRNA-lncRNA-mRNA feed-forward loops in Table IV, we evaluated the expression levels of 3 miRNA, 3 lncRNA, and 3 mRNA that are respectively located in 3 feed-forward loops. The average expression level of miR-127-5p was significantly increased, while miR-4786-5p and miR-297 were reduced in CLDN5-overexpressing hCMEC/D3 cells compared with control hCMEC/D3 cells. Analysis showed relatively high expression of miR-127-5p and low expression of n338895 and PIK3CG, and low expression of miR-4786-5p and miR-297 and high expression of n339695, TCONS_00022673-XLOC_010971 and CLDN1, OCLN (Fig. 4B). The 3 feed-forward loops detection by qRT-PCR are represented in Fig. 4C. 
A
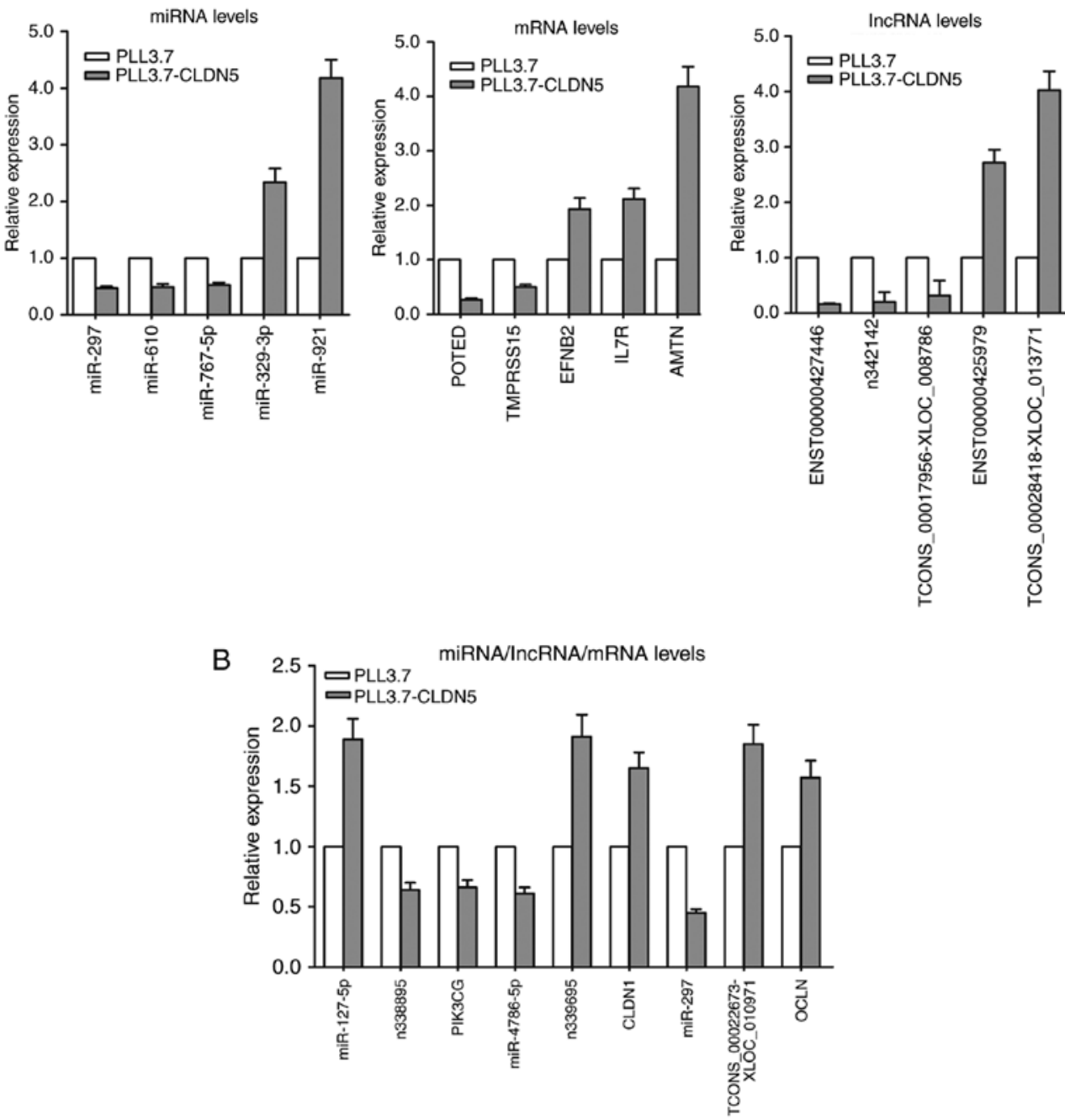

C

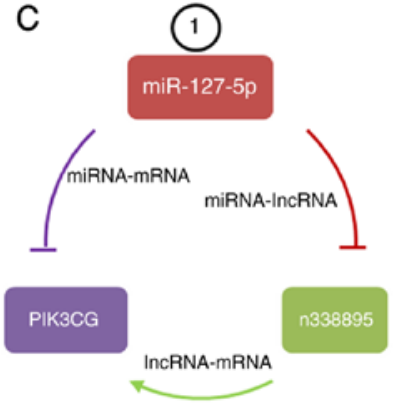

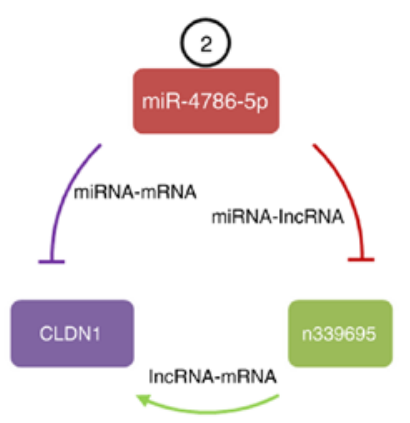

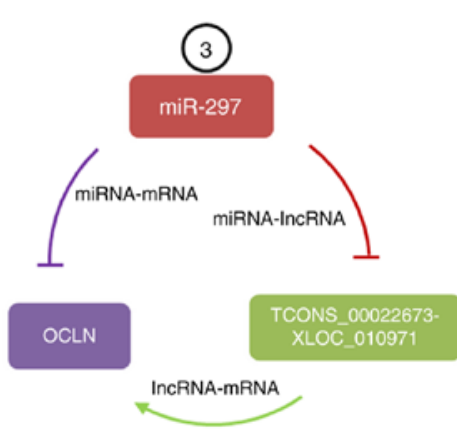

Figure 4. Expression analysis of miRNA, IncRNA, and mRNA in pLL3.7-CLDN5-transfected and pLL3.7 control hCMEC/D3 cells. The expression level of (A) 5 miRNAs, 5 lncRNAs and 5 mRNAs, (B) 3 miRNAs, 3 ncRNAs and 3 mRNAs that are respectively located in (C) 3 feed-forward-loops were analyzed using qRT-PCR, with the GAPDH (for lncRNA and mRNA) and $U 6$ (for miRNA) gene as an internal control. Error bars represent the standard errors of independent samples.

\section{Discussion}

In recent years, studies have confirmed the dysregulation of lncRNAs by acting as ceRNAs have profound implications for tumor metastasis. Reports demonstrate that lncRNAs as ceRNAs play an important role in blood-tumor barrier (BTB), and their dysfunction leads to the change of permeability of BTB. Several models have been proposed to explain how ceRNAs regulate BTB. Examples include lncRNA TUG1, NEAT1, XIST, MALAT1, which function in ceRNA manner to regulate expression of transcript factor (TF), such as
HSF2, SOX5, FOXC1/ZO-2 or nuclear factor (NF) NFYA through targeting different miR-144, miR-181d-5p, miR-137, and miR-140. These TFs and NF regulate the expression of claudin-5, occludin, and ZO-1, and then impact the permeability of BTB. Knockdown of lncRNAs TUG1, NEAT1, XIST, and MALAT1 resulted in increased the permeability of BTBs as well as decreased the expression of claudin-5, occludin, and ZO-1 (21-26). Although regulatory networks of ceRNA have now been shown to contribute to the permeability of BTB, the dysregulation of ceRNAs as a consequence of alterations in CLDN5 levels in BTB has not been previously investigated. 
In this study, we provide a comprehensive analysis of the roles of CLDN5 mediating the junctional and adhesion molecules, and signaling pathways through ceRNA interaction network in brain endothelial cells.

First of all, we used the Affymetrix GeneChip HTA 2.0 Array to analyze the distinct lncRNAs and mRNAs in CLDN5-overexpressing hCMEC/D3 cells. The sample matched miRNA expression profiling was determined by the Affymetrix GeneChip miRNA 4.0 Array. We identified a set of 41 miRNAs, 954 lncRNAs, and 222 mRNAs that differently expressed between pLL3.7-CLDN5-transfected and pLL3.7 control group. Such differentiation signified the potential roles of CLDN5 in cerebral vascular endothelial cells.

Furthermore, we discussed the effect of miRNA competition on the regulation of both IncRNAs and mRNAs, as well as the implications of IncRNA function as ceRNA through constructing the complex miRNA-lncRNA-mRNA interaction network. The possible biology functions of these regulatory ceRNAs mainly include tight junction, focal adhesion, cell-cell adhesion, cell growth and apoptosis. To our knowledge, this is the first study to show the lncRNA acting as ceRNAs were associated with clautin-5 function in brain vascular endothelial cells.

Our qRT-PCR expression analysis confirmed there are a series of miRNAs, IncRNAs, and mRNAs aberrantly expressed in the CLDN5-overexpressing hCMEC/D3 cells, which indicated that the differently expressed non-coding and coding RNAs might be characteristics regulated by CLDN5.

The expression of miR-127 was found to be upregulated, whereas miR-4786-5p and miR-297 were downregulated by CLDN5 overexpression. CLDN5 mediated interactions between metastasis tumor cells and brain endothelial cells. Recent studies showed miR-127 was aberrantly downregulated and acted as a functional tumor suppressor in several brain metastatic tumors, such as breast cancer (27), osteosarcoma (28), lung cancer (29), hepatocellular carcinoma (30), while miR-297 acts as an oncogene and is upregulated in lung adenocarcinoma and osteosarcoma $(31,32)$. Based on the findings obtained above, increased miR-127 and decreased miR-297 expression levels in claudin-5-overexpressing brain vascular endothelial cells can make BBB impermeable and reduce brain metastasis from cancer.

The PIK3CG, CLDN1 and OCLN mRNA regulated by ceRNA play key roles in the BBB. PIK3CG participates in the PI3K-Akt signaling pathway involved in regulation of numerous important cell processes including cell growth, differentiation, and metabolism. PI3K also was found to be among a couple of genes that were specifically altered in brain metastases of various tumor entities $(33,34)$, confirming previous reports that found specific alterations of the PI3K-Akt pathway in melanoma (35) and non-small lung cancer (36) brain metastases. The results of our study suggest that the PI3K-Akt pathway might be a promising target to prevent brain metastases by overexpression of claudin-5 through ceRNA. Occludin, claudin-1, and claudin- 5 are all TJ proteins, and they interact with other junctional proteins and play an important role in several pathologies, including tumor brain metastasis $(37,38)$. Our findings are particularly interesting in the dysregulated expression of CLDN1 and OCLN mRNA, in conjunction with the high CLDN5 levels.
Overall, our study identified and analyzed lncRNA function as ceRNA in CLDN5-overexpressing cerebral vascular endothelial cells, and in which ceRNA works as a downstream effector of the CLDN5 to strengthen several signaling pathways and mediate its role in maintaining BBB permeability. Understanding these ceRNA regulating networks would advance the development of prevention and therapy strategies for tumor brain metastasis.

Our findings indicated CLDN5 plays a key role in regulating BBB permeability through ceRNA network, so the activation of CLDN5 can inhibit tumor brain metastasis, which would be applied in medical treatment. Recent reports demonstrated that some proteases degrade tight junction protein, including CLDN5, and their inhibitor or antagonist prevents degration of tight junction protein and attenuates BBB disruption. Examples include telomerase $(39,40)$, and matrix metalloproteinases (MMPs) (41). Several models have been proposed to explain how telomerase regulated tight junction protein. First, telomerase activity may affect CLDN5; moreover AZT as an inhibitor of telomerase could modulate CLDN5 expression; at last, catalytic component of the telomerase holoenzyme may affect wnt signaling pathways (40). Earlier studies have shown that MMPs and their inhibitors-TIMPs play an essential role in the permission of drugs to cross the BBB $(42,43)$.

\section{Acknowledgements}

This study was supported by the National Natural Science Foundation of China (no. 81471229) and the Natural Science Foundation of Beijing City (nos. 7152098 and 7142054).

\section{Competing interests}

The authors declare that they have no competing interests.

\section{References}

1. Cardoso FL, Brites D and Brito MA: Looking at the blood-brain barrier: Molecular anatomy and possible investigation approaches. Brain Res Rev 64: 328-363, 2010.

2. Tam SJ and Watts RJ: Connecting vascular and nervous system development: Angiogenesis and the blood-brain barrier. Annu Rev Neurosci 33: 379-408, 2010.

3. Hawkins BT and Davis TP: The blood-brain barrier/neurovascular unit in health and disease. Pharmacol Rev 57: 173-185, 2005.

4. Bauer HC, Traweger A, Zweimueller-Mayer J, Lehner C, Tempfer H, Krizbai I, Wilhelm I and Bauer H: New aspects of the molecular constituents of tissue barriers. J Neural Transm (Vienna) 118: 7-21, 2011.

5. Eichler AF, Chung E, Kodack DP, Loeffler JS, Fukumura D and Jain RK: The biology of brain metastases-translation to new therapies. Nat Rev Clin Oncol 8: 344-356, 2011.

6. Furuse M, Fujita K, Hiiragi T, Fujimoto K and Tsukita S: Claudin-1 and -2: Novel integral membrane proteins localizing at tight junctions with no sequence similarity to occludin. J Cell Biol 141: 1539-1550, 1998.

7. Ohtsuki S, Yamaguchi H, Katsukura Y, Asashima $\mathrm{T}$ and Terasaki T: mRNA expression Levels of tight junction protein genes in mouse brain capillary endothelial cells highly purified by magnetic cell sorting. J Neurochem 104: 147-154, 2008.

8. Nitta T, Hata M, Gotoh S, Seo Y, Sasaki H, Hashimoto N, Furuse $M$ and Tsukita S: Size-selective loosening of the blood-brain barrier in claudin-5-deficient mice. J Cell Biol 161: 653-660, 2003.

9. Ouban A and Ahmed AA: Claudins in human cancer: A review. Histol Histopathol 25: 83-90, 2010. 
10. Zamore PD and Haley B: Ribo-gnome: The big world of small RNAs. Science 309: 1519-1524, 2005.

11. Guttman M, Amit I, Garber M, French C, Lin MF, Feldser D, Huarte M, Zuk O, Carey BW, Cassady JP, et al: Chromatin signature reveals over a thousand highly conserved large non-coding RNAs in mammals. Nature 458: 223-227, 2009.

12. Salmena L, Poliseno L, Tay Y, Kats L and Pandolfi PP: The ceRNA hypothesis: The rosetta stone of a hidden RNA language. Cell 146: 353-358, 2011

13. Karreth FA, Tay Y, Perna D, Ala U, Tan SM, Rust AG, DeNicola G, Webster KA, Weiss D, Perez-Mancera PA, et al: In vivo identification of tumor-suppressive PTEN ceRNAs in an oncogenic BRAF-induced mouse model of melanoma. Cell 147: 382-395, 2011

14. Tay Y, Kats L, Salmena L, Weiss D, Tan SM, Ala U, Karreth F, Poliseno L, Provero P, Di Cunto F, et al: Coding-independent regulation of the tumor suppressor PTEN by competing endogenous mRNAs. Cell 147: 344-357, 2011.

15. Wang J, Liu X, Wu H, Ni P, Gu Z, Qiao Y, Chen N, Sun F and Fan Q: CREB up-regulates long non-coding RNA, HULC expression through interaction with microRNA-372 in liver cancer. Nucleic Acids Res 38: 5366-5383, 2010.

16. Fan M, Li X, Jiang W, Huang Y, Li J and Wang Z: A long non-coding RNA, PTCSC3, as a tumor suppressor and a target of miRNAs in thyroid cancer cells. Exp Ther Med 5: 1143-1146, 2013.

17. Panzitt K, Tschernatsch MM, Guelly C, Moustafa T, Stradner M, Strohmaier HM, Buck CR, Denk H, Schroeder R, Trauner M and Zatloukal K: Characterization of HULC, a novel gene with striking up-regulation in hepatocellular carcinoma, as noncoding RNA. Gastroenterology 132: 330-342, 2007.

18. Jendrzejewski J, He H, Radomska HS, Li W, Tomsic J, Liyanarachchi S, Davuluri RV, Nagy R and de la Chapelle A: The polymorphism rs 944289 predisposes to papillary thyroid carcinoma through a large intergenic noncoding RNA gene of tumor suppressor type. Proc Natl Acad Sci 109: 8646-8651, 2012.

19. Johnsson P, Ackley A, Vidarsdottir L, Lui WO, Corcoran M, Grandér D and Morris KV: A pseudogene long-noncoding-RNA network regulates PTEN transcription and translation in human cells. Nat Struct Mol Biol 20: 440-446, 2013.

20. Prieto C, Risueño A, Fontanillo C and De las Rivas J: Human gene coexpression landscape: Confident network derived from tissue transcriptomic profiles. PLoS One 3: e3911, 2008.

21. Cai H, Xue Y, Wang P, Wang Z, Li Z, Hu Y, Li Z, Shang X and Liu Y: The long noncoding RNA TUG1 regulates blood-tumor barrier permeability by targeting miR-144. Oncotarget 6 : 19759-19779, 2015.

22. Zhao L, Wang P, Liu Y, Ma J and Xue Y: miR-34c Regulates the permeability of blood-tumor barrier via MAZ-mediated expression changes of ZO-1, Occludin, and Claudin-5. J Cell Physiol 230: 716-731, 2015.

23. Miao YS, Zhao YY, Zhao LN, Wang P, Liu YH, Ma J and Xue YX: MiR-18a increased the permeability of BTB via RUNX1 mediated down-regulation of ZO-1, occludin and claudin-5. Cell Signal 27: 156-167, 2015.

24. Guo J, Cai H, Zheng J, Liu X, Liu Y, Ma J, Que Z, Gong W, Gao Y, TaoW and Xue Y:Long non-coding RNANEAT1 regulates permeability of the blood-tumor barrier via miR-181d-5p-mediated expression changes in ZO-1, occludin, and claudin-5. Biochim Biophys Acta 1863: 2240-2254, 2017.

25. Yu H, Xue Y, Wang P, Liu X, Ma J, Zheng J, Li Z, $\mathrm{Li} \mathrm{Z}$, Cai $\mathrm{H}$ and Liu Y: Knockdown of long non-coding RNA XIST increases blood-tumor barrier permeability and inhibits glioma angiogenesis by targeting miR-137. Oncogenesis 6: e303, 2017.

26. Ma J, Wang P, Yao Y, Liu Y, Li Z, Liu X, Li Z, Zhao X, Xi Z, Teng $\mathrm{H}$, et al: Knockdown of long non-coding RNA MALAT1 increases the blood-tumor barrier permeability by up-regulating miR-140. Biochim Biophys Acta 1859: 324-338, 2016.
27. Wang S, Li HJ, Wang J, Wang D, Yao A and Li Q: Prognostic and biological significance of microRNA-127 expression in human breast cancer. Dis Markers 2014: 401986, 2014.

28. Zhang J, Hou W, Chai M, Zhao H, Jia J, Sun X, Zhao B and Wang R: MicroRNA-127-3p inhibits proliferation and invasion by targeting SETD8 in human osteosarcoma cells. Biochem Biophys Res Commun 469: 1006-1011, 2016.

29. Shi L, Wang Y, Lu Z, Zhang H, Zhuang N, Wang B, Song Z Chen G, Huang C, Xu D, et al: miR-127 promotes EMT and stem-like traits in lung cancer through a feed-forward regulatory loop. Oncogene 36: 1631-1643, 2017.

30. Huan L, Bao C, Chen D, Li Y, Lian J, Ding J, Huang S, Liang L and $\mathrm{He} \mathrm{X}$ : MicroRNA-127-5p targets the biliverdin reductase $\mathrm{B} /$ nuclear factor- $\kappa \mathrm{B}$ pathway to suppress cell growth in hepatocellular carcinoma cells. Cancer Sci 107: 258-266, 2016.

31. Sun Y, Zhao J, Yin X, Yuan X, Guo J and Bi J: miR-297 acts as an oncogene by targeting GPC5 in lung adenocarcinoma. Cell Prolif 49: 636-643, 2016.

32. Wang Y and Kong D: Knockdown of lncRNA MEG3 inhibits viability, migration, and invasion and promotes apoptosis by sponging miR-127 in osteosarcoma cell. J Cell Biochem 119: 669-679, 2018.

33. Bai Y, Zhang Y, Hua J, Yang X, Zhang X, Duan M, Zhu X, Huang W, Chao J, Zhou R, et al: Silencing microRNA-143 protects the integrity of the blood-brain barrier: Implications for methamphetamine abuse. Sci Rep 6: 35642, 2016.

34. Brastianos PK, Carter SL, Santagata S, Cahill DP, TaylorWeiner A, Jones RT, Van Allen EM, Lawrence MS, Horowitz PM, Cibulskis K, et al: Genomic characterization of brain metastases reveals branched evolution and potential therapeutic targets. Cancer Discov 5: 1164-1177, 2015.

35. Chen G, Chakravarti N, Aardalen K, Lazar AJ, Tetzlaff MT, Wubbenhorst B, Kim SB, Kopetz S, Ledoux AA, Gopal YN, et al: Molecular profiling of patient-matched brain and extracranial melanoma metastases implicates the PI3K pathway as a therapeutic target. Clin Cancer Res 20: 5537-5546, 2014.

36. Li Q, Yang J, Yu Q, Wu H, Liu B, Xiong H, Hu G, Zhao J, Yuan X and Liao Z: Associations between single-nucleotide polymorphisms in the PI3K-PTEN-AKT-mTOR pathway and increased risk of brain metastasis in patients with non-small cell lung cancer. Clin Cancer Res 19: 6252-6260, 2013.

37. Piontek J, Winkler L, Wolburg H, Müller SL, Zuleger N, Piehl C, Wiesner B, Krause G and Blasig IE: Formation of tight junction: Determinants of homophilic interaction between classic claudins. FASEB J 22: 146-158, 2008.

38. Wilhelm I, Molnár J, Fazakas C, Haskó J and Krizbai IA: Role of the blood-brain barrier in the formation of brain metastases. Int J Mol Sci 14: 1383-1411, 2013.

39. Huang W, Rha GB, Chen L, Seelbach MJ, Zhang B, András IE, Bruemmer D, Hennig B and Toborek M: Inhibition of telomerase activity alters tight junction protein expression and induces transendothelial migration of HIV-1-infected cells. Am J Physiol Heart Circ Physiol 298: H1136-1145, 2010.

40. Armando RG, Gomez DM and Gomez DE: AZT exerts its antitumoral effect by telomeric and non-telomeric effects in a mammary adenocarcinoma model. Oncol Rep 36: 2731-2736, 2016.

41. Yang Y and Rosenberg GA: MMP-mediated disruption of claudin-5 in the blood-brain barrier of rat brain after cerebral ischemia. Methods Mol Biol 762: 333-345, 2011.

42. De Lorenzo MS, Alonso DF and Gomez DE: Nafoxidine modulates the expression of matrix-metalloproteinase-2 (MMP-2) and tissue inhibitor of metalloproteinases-1 (TIMP-1) in endothelial cells. Anticancer Res 20(1A): 395-400, 2000

43. Cyr M, Calon F, Morissette M and Di Paolo T: Estrogenic modulation of brain activity: implications for schizophrenia and Parkinson's disease. J Psychiatry Neurosci 27: 12-27, 2002. 